\title{
PREDICTING THE ELASTO-PLASTIC RESPONSE OF SHORT FIBER COMPOSITES USING DEEP NEURAL NETWORKS TRAINED ON MICRO-MECHANICAL SIMULATIONS
}

\author{
$\underline{\text { J. Friemann }}^{1 *}$, B. Dashtbozorg ${ }^{2}$, M. Fagerström ${ }^{1}$ and S.M Mirkhalaf ${ }^{3}$ \\ ${ }^{1}$ Department of Industrial and Materials Science, Chalmers University of Technology, Gothenburg, \\ Sweden \\ ${ }^{2}$ Department of Biomedical Engineering, Eindhoven University of Technology, Eindhoven, the \\ Netherlands \\ ${ }^{3}$ Department of Physics, University of Gothenburg, Gothenburg, Sweden \\ *johan.friemann@outlook.com
}

The mechanical modeling of Short Fiber Reinforced Composites (SFRC) still poses several challenges. This is partly owing to the high degree of anisotropy, and the discontinuity and orientation distribution of fibers. Being able to accurately predict the behavior of SFRC with varying fiber orientations and fiber volume fractions is highly relevant in the design and production of injection molded parts. It has therefore become popular to abandon classical constitutive models in favor of data driven models [1,2].

The training of an Artificial Neural Network (ANN) is a popular and efficient method of using large amounts of data to teach an algorithm the underlying rules of a phenomenon, enabling it to make predictions for data it has never before encountered. ANNs have successfully been utilized to describe the elastic properties of SFRC with arbitrary fiber orientations [3]. However, a model that handles path-dependent plasticity in 3D in an SFRC with arbitrary fiber orientations is yet to be developed.

In this work, a recurrent deep neural network model utilizing Gated Recurrent Units is developed to predict the elasto-plastic stress-strain response of a short fiber composite material. The model is designed to have the ability to make predictions for arbitrary fiber orientations and varying fiber volume fractions. The training data is generated by performing micro-mechanical simulations utilizing mean field methods in the commercially available software DIGIMAT-MF. Special concern is taken to construct a very general training set, such that the model can be trained on a large variety of strain paths. For this purpose we propose to generate the strain input through a novel random walk scheme in the strain space. The numerical results show that the proposed model performs well, and the mean error for a typical load cycle usually stays below $10 \%$ of the matrix yield stress.

\section{References}

[1] M. Mozaffar, R. Bostanabad, W. Chen, K. Ehmann, J. Cao, and M. A. Bessa. Deep learning predicts path-dependent plasticity. Proceedings of the National Academy of Sciences, 116(52):26414-26420, December 2019.

[2] A. Zhang and D. Mohr. Using neural networks to represent von Mises plasticity with isotropic hardening. International Journal of Plasticity, 132, 2020.

[3] N. Mentges, B. Dashtbozorg, and S.M. Mirkhalaf. A micromechanics-based artificial neural networks model for elastic properties of short fiber composites. Composites Part B: Engineering, 2021:108736. 